\title{
Features of Acanthocephalan Hooks Using Dual Beam Preparation and XEDS Phase Maps
}

\author{
Michael D. Standing ${ }^{1}$, Richard A. Heckmann ${ }^{2}$ \\ ${ }^{1}$ Microscopy Lab, Brigham Young University, Provo, Utah, United States of America \\ ${ }^{2}$ Department of Biology, Brigham Young University, Provo, Utah, United States of America
}

Acanthocephala is a minor phylum of dioecious worms (Helminths) which is often overlooked in beginning biology and parasitology courses [1]. Hooks are a major characteristic of all known species, thus they have been referred to as thorny headed or spiny headed worms. An evertable (protrudible) proboscis is armed with the hooks which provide permanent attachment to the host intestine. Over 1150 species have been described with new ones listed each year. Phylogenetically, based on DNA studies, their nearest relatives are not other worms (nematodes, cestodes) but rotifers. They range in size from microscopic to $65 \mathrm{~mm}$. Fish and birds are common hosts while only eight species have been isolated from humans to date [2].

The hooks of the Acanthocephala, a major key for classification, may be of various shapes, sizes, and number and by muscular contractions can migrate thru the intestinal layers into the abdominal cavity of its host [3]. There is limited information about the chemical nature and formation of the hooks. Electron microscopy has added an essential part to understanding the spiny headed worms especially the hooks. Current studies based on morphology and elemental content relate these hooks to human and other mammalian hard tissues. Previous studies have shown the presence of calcium, sulfur, and phosphorous $[4]$.

Observing hook morphology using conventional scanning electron microscopy (SEM) yields the general shape, arrangement, and surface structure of the hooks. Nothing about the internal structure of the hooks can be obtained using SEM unless a hook happens to be broken. Transmission electron microscopy of the hooks can be performed, but orientation of the hooks is difficult. Using a dual beam instrument to provide a longitudinal section of an individual hook provides access to visualize the internal morphology of a hook and its origin, and to perform more specific elemental x-ray analysis (XEDS) of the hook than can be obtained from its exterior surface.

Samples of Acanthocephala were obtained that were allowed to relax in water (to encourage proboscis eversion) and then preserved in 70\% ETOH for storage. Samples were rehydrated and post fixed in 1\% buffered $\mathrm{OsO}_{4}$ for 2 hours, and then dehydrated in an acetone series and critical point dried. Samples were then mounted on SEM sample mounts using double-sided carbon tape, sputter coated with $15 \mathrm{~nm}$ AuPd, and observed in a FEI Helios Nanolab 600. Suitable hooks were located, cross-sectioned with the $\mathrm{Ga}^{+}$beam, imaged, and then analyzed using an EDAX XEDS system running their TEAM analytical software. Morphologic analysis showed the hook attachment in the proboscis while XEDS phase maps and elemental maps showed strong calcium and phosphorous concentrations in the interior of the hook with a sulfur rich sheath surrounding the calcified area. Use of a dual beam instrument has shown to be an instrumental method for obtaining useful information about the interior structures and chemistry of small, hard, difficult to orient biological structures. 
[1] Roberts, L.S., and J. Janovy Jr., "Foundations of Parasitology $8^{\text {th }}$ ed.” McGraw-Hill, 502p.

[2] Hausten, T. et al, Clinical Microbiology and Infection, vol. 16, 2009, p.6

[3] Amin, O.M.; Folia Parasitologica, vol. 16, 2013, p. 273

[4] Heckmann, R.A. et al, Comparative Parasitology, vol. 74, 2007, p. 388
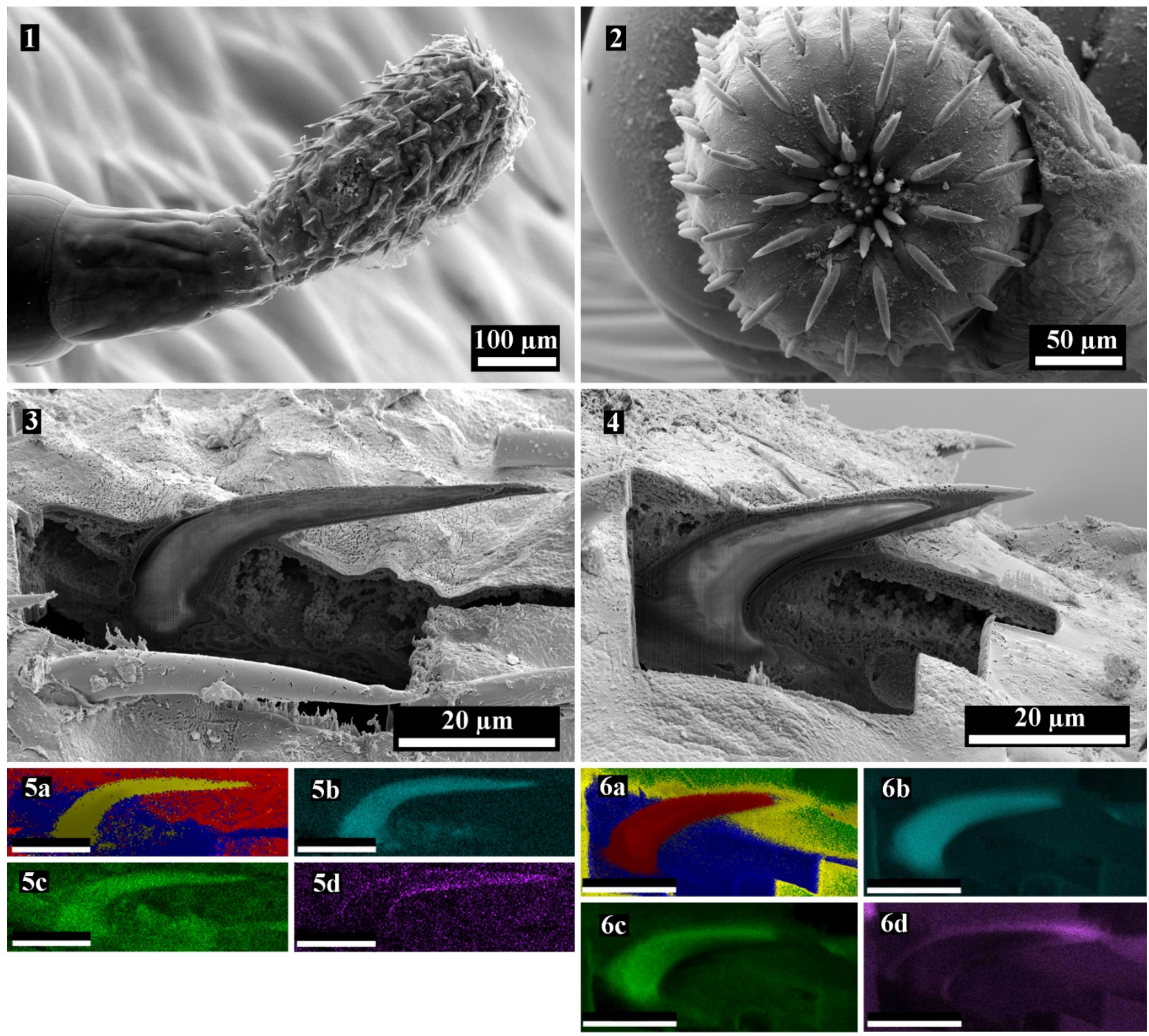

Figure 1: Lateral view of Trajectura sp.

Figure 2: End-on view of Echinoryhnchus sp.

Figure 3: Ion-milled longitudinal cross section of Trajectura sp. hook.

Figure 4: Ion-milled longitudinal cross-section of Echinorynchus sp. hook.

Figures 5 and 6: X-ray maps from the respective hooks imaged above. Bar $=20 \mu \mathrm{m}$ A) Phase map showing the various chemistries of the hook and surrounding area. Each color represents a different chemistry. B) Calcium x-ray map. C) Phosphorous x-ray map. D) Sulfur x-ray map. 